\title{
The relative timing of supra-arcade downflows in solar flares
}

\author{
J. I. Khan, H. M. Bain, and L. Fletcher
}

Department of Physics and Astronomy, University of Glasgow, Glasgow G12 8QQ, Scotland, UK

e-mail: jkhan@spd.aas.org

Received 14 May 2007 / Accepted 11 August 2007

\section{ABSTRACT}

\begin{abstract}
Context. Supra-arcade downflows (generally dark, sunward-propagating features located above the bright arcade of loops in some solar flares) have been reported mostly during the decay phase, although some have also been reported during the rise phase of solar flares.

Aims. We investigate, from a statistical point of view, the timing of supra-arcade downflows during the solar flare process, and thus determine the possible relation of supra-arcade downflows to the primary or secondary energy release in a flare.

Methods. Yohkoh Soft X-ray Telescope (SXT) imaging data are examined to produce a list of supra-arcade downflow candidates. In many of our events supra-arcade downflows are not directly observed. However, the events do show laterally moving (or "waving") bright rays in the supra-arcade fan of coronal rays which we interpret as due to dark supra-arcade downflows. The events are analysed in detail to determine whether the supra-arcade downflows (or the proxy waving coronal rays) occur during a) the rise and/or decay phases of the soft X-ray flare and b) the flare hard X-ray bursts. It is also investigated whether the supra-arcade downflows events show prior eruptive signatures as seen in SXT, other space-based coronal data, or reported in ground-based $\mathrm{H} \alpha$ images.

Results. A substantial majority of supra-arcade downflow events show downflows which start during the soft X-ray flare rise phase (73\%), occur during hard X-ray bursts (90\%), and have prior eruptive signatures associated with them (73\%). However, we find a single event ( $2 \%$ of the total) which clearly and unambiguously showed supra-arcade downflows starting during the soft X-ray flare decay phase.

Conclusions. Since the majority of supra-arcade downflows occur during the rise phase of the soft X-ray flare and the time of hard X-ray bursts, and have prior eruptive signatures this suggests that they are related to the main flare energy release process. Furthermore, the suggested association of supra-arcade downflows with recently reconnected magnetic field lines means they may indeed be considered as evidence for a magnetic reconnection process. The single supra-arcade downflow event which unambiguously started during the decay phase of the flare occurred during hard X-ray bursts and thus appears to be related to late energy release.
\end{abstract}

Key words. Sun: corona - Sun: coronal mass ejections (CMEs) - Sun: flares - Sun: magnetic fields - Sun: X-rays, gamma rays magnetohydrodynamics (MHD)

\section{Introduction}

Soft X-ray and EUV images of some solar flares have revealed the presence of sunward-propagating features located above the bright flaring arcade of loops (McKenzie \& Hudson 1999, 2001; McKenzie 2000; Gallagher et al. 2002; Innes et al. 2003a; Sheeley et al. 2004; Asai et al. 2004). In soft X-ray images these supra-arcade downflows generally appear as dark blob-like features occurring in dark channels between soft X-ray emitting rays which are located above the bright flaring arcade of closed loops. The bright ray-like structure, first reported in soft X-ray images by Švestka et al. (1998), has been referred to simply as a fan of coronal rays. The arcade plus fan of coronal rays have also been referred to collectively as "spiky arcades" (Hudson $\&$ McKenzie 2001). However we prefer to use the term "spiny arcades" to avoid confusion, as spikes and spiky behaviour are used in other contexts in solar physics and to describe quite different phenomena. Supra-arcade downflows (and the fan of coronal rays) observed in soft X-ray imaging data have mostly been reported as occurring during the decay phase of flares. A few events were also reported as showing downflows during the rise phase of the flares (McKenzie 2000), although the significance of this was not stressed at the time. The projected plane-ofsky propagation speeds for these supra-arcade downflowing features have been determined to be in the range $\sim 35-500 \mathrm{~km} \mathrm{~s}^{-1}$
(McKenzie \& Hudson 2001). As far as we are aware 17 supraarcade downflow events have been reported using Yohkoh Soft X-ray Telescope (SXT) (Tsuneta et al. 1991) data (McKenzie \& Hudson 1999, 2001; McKenzie 2000; Grechnev et al. 2006).

To the best of our knowledge, to date, only 3 supra-arcade downflow events have been reported in EUV observations from the Transition Region and Coronal Explorer (TRACE) (Handy et al. 1999; Gallagher et al. 2002; Innes et al. 2003a,b; Sheeley et al. 2004; Asai et al. 2004). In TRACE EUV images the supra-arcade downflows appear as sunward propagating dark regions with laterally moving (i.e., "waving" or "wriggling") trailing elongated dark regions giving rise to a tadpole-like appearance (Verwichte et al. 2005). A bright fan of coronal rays is not seen in the TRACE $195 \AA$ filter images. Instead an apparently amorphous region located above the bright flaring arcade of closed loops is seen in emission into which the dark downflows appear to penetrate. This emission is likely to be due to the Fe XXIV $192 \AA$ resonance line lying within the TRACE $195 \AA$ filter passband, indicating the presence of plasma with temperatures $\gtrsim 15$ MK (Phillips et al. 2005). Asai et al. (2004) demonstrated that the TRACE EUV supra-arcade downflows for the single event they considered occurred during the rise phase of the flare (as well as later in the flare) and the times of the downflows appeared to correlate well with the times of hard X-ray bursts. 
Spectroscopic studies of supra-arcade downflows by Innes et al. (2003a), show that the dark channels associated with them are generally dark in lines sensitive to plasmas over the temperature range $10^{4}-10^{7} \mathrm{~K}$, suggesting they are due to voids in the plasma, rather than colder absorbing plasma (e.g., as is the case for dark $\mathrm{H} \alpha$ filaments). This is also often the case for the front of the dark channels. However, in one case, Innes et al. (2003a) did find evidence for absorption at the front of the inflow suggesting cooler plasma.

Bright supra-arcade downflowing features and bright shrinking loops have also been reported during flares (McKenzie 2000) Downflows recently reported by Tripathi et al. (2006) appear to be a different type of phenomenon, probably associated with a falling prominence/filament. Recently, Khan et al. (2006) reported observations of simultaneous retracting and expanding distinct coronal loops observed in emission in soft X-rays during a solar flare. For the particular event they examined, there were also supra-arcade downflows and these were clearly observed to start during the decay phase of the flare.

Švestka et al. (1998) suggested that the supra-arcade coronal rays are outflowing plasma. In recent work, Verwichte et al. (2005) used the suggestion that the supra-arcade coronal rays are outflowing jets of plasma to develop a model to understand the downflows. They concluded that the bright outflowing rays lead to apparent dark downflows in the regions between them.

The time during which supra-arcade downflows generally occur in relation to the stages in the flare process is not yet clear from previous work. There is, consequently, a clear need to establish the timing of supra-arcade downflows relative to stages in the flare process from a statistical point of view, using a systematic study of many events. This is important for understanding physical processes which may give rise to supra-arcade downflows, as well as their significance in the flare process. For example, in the widely suggested scenario for supra-arcade downflows which associates them with retracting reconnected loops (essentially void of plasma) the supra-arcade downflows should, in principle, be associated with the main flare energy release, i.e., the flare rise phase (and possibly later if there is continued energy release). However it has not yet been determined if this is indeed the case for most supra-arcade downflow events. In the scenario proposed by Verwichte et al. (2005) supra-arcade downflows presumably need not occur during the flare rise phase.

In this paper we examine many supra-arcade downflow events, determine their timing and compare these with the times of the soft X-ray flare rise and decay intervals and (flare) hard $\mathrm{X}$-ray bursts. We also investigate whether supra-arcade downflow events are associated with eruptive signatures as seen in SXT images, other space-based coronal data, or in $\mathrm{H} \alpha$ images. The sources of data used in this work and the criteria used to select supra-arcade downflow events are outlined in Sect. 2. In Sect. 3 we present example events to illustrate how we constructed our table of events. In Sect. 4 we present the results of our analysis. Conclusions are summarised in Sect. 5 where a brief discussion of our results is also given.

\section{Data sources and event selection}

\subsection{The sources of data used in this study}

In this paper we use the following data:

- Lists of flares observed by the soft X-ray detectors on board the Geostationary Operational Environmental Satellite (GOES). These lists are prepared by the USA
National Oceanic and Atmospheric Administration (NOAA) Space Environment Center (SEC).

- Observing lists of soft X-ray images from the Yohkoh SXT. These are obtained using standard SolarSoft software for Yohkoh analysis and the standard Yohkoh database files.

- Yohkoh flare-mode and quiet-mode partial-frame images (PFIs).

- Whole-Sun soft X-ray fluxes from detectors on board GOES. These cover the wavelength ranges $\sim 0.5-4 \AA$ and $\sim 1-8 \AA$.

- Whole-Sun hard X-ray fluxes from the Yohkoh Hard X-ray Telescope (HXT) (Kosugi et al. 1991) as well as the Discriminator Large Area (DISCLA) detectors on the Burst and Transient Source Experiment (BATSE) on board the Compton Gamma-ray Observatory (Fishman et al. 1989).

- Whole-Sun $195 \AA$ images from the Solar and Heliospheric Observatory (SOHO) Extreme ultraviolet Imaging Telescope (EIT) (Delaboudinière et al. 1995).

- SOHO Large Angle Spectroscopic Coronagraph (LASCO) data (Brueckner et al. 1995).

- Lists of prominences and other eruptive signatures from the publication Solar Geophysical Data.

\subsection{The selection of supra-arcade downflow events}

We obtained our list of supra-arcade downflow events as follows. We constructed a list of time intervals for which there are flaremode partial-frame images from SXT using the Yohkoh observing logs for the period 1991 October 01-2001 December 14, inclusive. (In order to see the supra-arcade downflows we believe we require the higher cadence flare-mode observations). We compared this SXT list with a list of GOES soft X-ray flares obtained from catalogues prepared by the SEC using SolarSoft and Interactive Data Language (IDL) software. We only retained the Yohkoh SXT list entries if there was a temporally overlapping $G O E S$ flare entry (this was done to avoid false flares observed by Yohkoh).

A few comments are necessary concerning the GOES soft X-ray flare times in the SEC lists and our tabulated estimates. Note that for GOES flares prior to 1997 the flare start times in these lists are for the optical $(\mathrm{H} \alpha)$ flare, if there was a corresponding optical event. Also the flare end time is defined to be the time the soft X-ray flux reaches $0.5 \times$ (the flare peak flux + flux at the start of the flare). Thus the SEC GOES flare end time will not represent a time close to that when the flare actually ended (e.g., where the flux returned to a level near to that prior to the flare or to a new background level). To avoid rejecting some Yohkoh flare-mode data observed late in the decay phase of some flares (i.e., occurring after the SEC-listed "end" of the GOES soft X-ray flare) we used the following method. We searched for SXT flare-mode data from the earlier of the GOES flare start and SXT flare-mode start until 1 minute before the start of the next GOES tabulated flare. With this selection method we find 3579 Yohkoh flare-mode flares which overlapped GOES soft X-ray flares.

Previous work by McKenzie \& Hudson (1999, 2001); McKenzie (2000) found that supra-arcade downflow events were best observed in SXT half-resolution (4.91" pixel) flaremode PFIs. A quick look at several events already reported and some new events we have recently discovered reveal that those images are indeed likely to be the most appropriate for finding such events. Since supra-arcade downflows are faint and require over-exposed images, the SXT full resolution (2.45" pixel) flare-mode PFIs, which generally do not have overexposed images, are very unlikely to show such features. While 
supra-arcade downflows have been observed in SXT quarter resolution (9.82" pixel) flare-mode PFIs, the coarser spatial resolution makes them more difficult to observe. Consequently, we further restricted our list of SXT data to those which had halfresolution (4.91" pixel) data. This reduces the number of SXT flares to 3114. It may be possible that some supra-arcade downflow events could be observed in quarter-resolution images, but not in half-resolution images. However, we believe that we will not lose many events with our half-resolution image criterion.

Previously reported supra-arcade downflow events in the Yohkoh SXT images by McKenzie \& Hudson (1999, 2001), McKenzie (2000), Grechnev et al. (2006) and in TRACE EUV images have been, with two exceptions, M- or X-class flares. While C-class or even lower peak flux flares may also show supra-arcade downflows, M- or X-class flares will probably show them more clearly. Thus we further restricted the list of SXT flares by retaining only those identified as corresponding to M- or X-class GOES soft X-ray flares. With this additional restriction we end up with the following flares to search for supraarcade downflow events: $69 \mathrm{X}$-class flares, 115 flares in the range M4.0-9.9 class, and 682 flares in the range M1.0-3.9 class.

After drawing up these lists we then examined the corrected flare-mode, half-resolution SXT PFI data for these events. The initial selection procedure involved noting those events which appeared to show supra-arcade downflows or a supra-arcade fan of coronal rays in the half-resolution, flare-mode PFIs. Next we examined each of these supra-arcade downflow candidate events using all available SXT PFI data, i.e., flare-mode and quiet-mode PFIs of all resolutions, to determine the start and end times of the supra-arcade downflows. We did not examine the whole-Sun full-frame images (FFIs) for such events because the cadence of the images (generally $\sim 10 \mathrm{~min}$ ) is too low to show supra-arcade downflows.

In many events, distinct and apparently downflowing blobs could not always be seen. However, the supra-arcade fan of coronal rays would generally show a marked lateral motion ("waving" or "wriggling") which we interpret as due to downflows. The justification for this is based on our observational finding that events which clearly show supra-arcade downflows in the SXT data also show "waving coronal rays" clearly associated with the downflows. This is also apparent in the movies CD-ROM accompanying the article McKenzie (2000) and the data presented by Verwichte et al. (2005). Consequently, in all of our events when determining the timing of the supra-arcade downflows we used either supra-arcade downflowing blobs or a lateral motion of the supra-arcade fan of coronal rays (treating it as a proxy for supra-arcade downflowing blobs). We find 41 supra-arcade downflow events in the SXT data using this procedure.

We also examined both Yohkoh HXT (13-93 keV) and BATSE DISCLA $25-50 \mathrm{keV}$ and $50-100 \mathrm{keV}$ data to determine whether hard X-ray bursts were observed during these flares. If so, the interval they occurred was noted. This was then compared with the time supra-arcade downflows were seen. For this statistical study we do not consider detailed comparisons between the times of the downflow features and the times of hard X-ray bursts, as was done by Asai et al. (2004), nor do we consider hard X-ray imaging data. We simply take a temporal overlap to indicate a possible relation between the supra-arcade downflows and the hard X-ray bursts.

Our list of 41 events and various time intervals we determined are shown in Table 1 . In this table we also indicate whether the supra-arcade downflows occurred during the rise or decay phases of the GOES soft X-ray flares and during the time interval of hard X-ray bursts. While examining the SXT data we noted if the events showed prior eruptive soft X-ray signatures. That is, we checked if the SXT data showed the eruption of a soft X-ray emitting coronal loop prior to, and in the general vicinity of, the supra-arcade downflows. We also examined SOHO EIT $195 \AA$ Amages and SOHO LASCO data for evidence of (interpolated) prior eruptive signatures such as coronal mass ejections or filament eruptions. Furthermore, we also examined lists of prominences and other eruptive signatures as reported in Solar Geophysical Data. We took evidence for the eruption of a large-scale loop as an eruptive signature. In the last column in the table if a prior eruptive signature was found we indicate one of the data sources for that evidence. SXT, EIT, LASCO indicate an eruption is seen in the SXT, EIT and LASCO data, respectively. EPL indicates an eruptive prominence on the solar limb was reported in $\mathrm{H} \alpha$ data to Solar Geophysical Data.

The SEC lists of GOES soft X-ray flares give flare times only to the nearest minute and, as already mentioned, have flare end times which are not adequate for our purposes. We examined the GOES $\sim 3 \mathrm{~s} \sim 1-8 \AA$ data and determined our best estimate for the times of the associated GOES soft X-ray flare for each event. In some events there are multiple overlapping GOES flares. We carefully examined the SXT and GOES data to determine the appropriate GOES soft X-ray flare. In some cases we find that the particular flare associated with the supra-arcade downflows was different from the nominally assigned soft X-ray class (usually the peak in an overlapping sequence). We do in fact find two of the events are associated with C-class flares, which we nonetheless retain. The GOES flare intervals are given in Table 1, which we estimate are accurate to $\sim \pm 10 \mathrm{~s}$. We also determined the location of the flare from the SXT data and SEC lists of $\mathrm{H} \alpha$ flares.

\section{Example supra-arcade downflow events}

In order to demonstrate how we constructed the table of entries, and the different types of events we found, we now present example supra-arcade downflow events. Note that we define the terms flare rise phase and decay phase to be the intervals from GOES soft X-ray flare start to peak and from GOES soft X-ray flare peak to end, respectively.

\subsection{Downflows starting during the flare rise phase}

We now show an example of an event with good temporal coverage during the flare rise phase and for which we find supraarcade downflows clearly started during the rise phase and overlapped hard X-ray emission.

We consider the event on 2000 November 25. The GOES soft $\mathrm{X}$-ray flux for this flare is shown in Fig. 1. Solid-hatched intervals on the plot indicate when Yohkoh was behind the Earth (and did not observe the Sun), while the dotted-hatched intervals indicate when it passed through the South Atlantic Anomaly region. Tick marks (some of which appear as bands) at the bottom of the plot indicate the times of SXT imaging data examined in this analysis. From this figure it can be seen that Yohkoh observed the rise phase and peak of this flare.

Examination of the SXT flare-mode data shows the supraarcade downflows or fan of waving coronal rays starting near $\sim 01: 15: 59$ UT and visible until 02:21:17 UT. In Fig. 2a we show a half-resolution (4.91" pixel) SXT image with a superposed full-resolution (2.45" pixel) image portion before the supra-arcade downflows are seen, but when erupting soft X-ray 


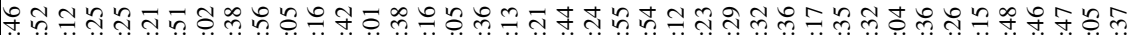

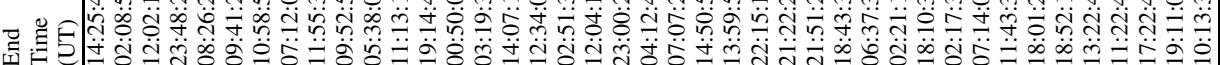

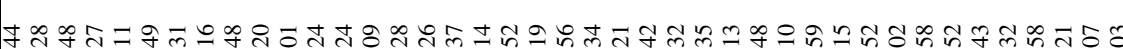

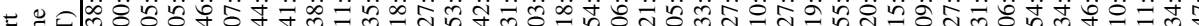

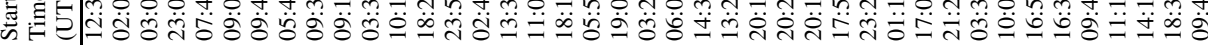

유구숬

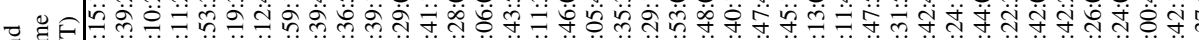

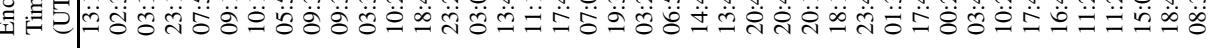

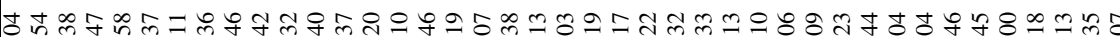
年

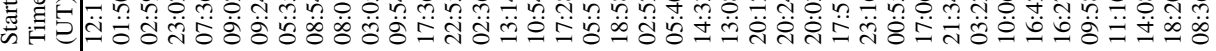

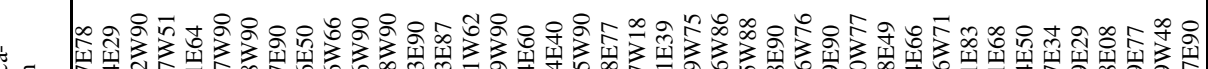

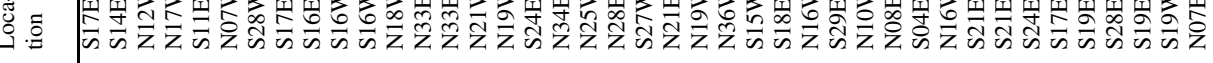

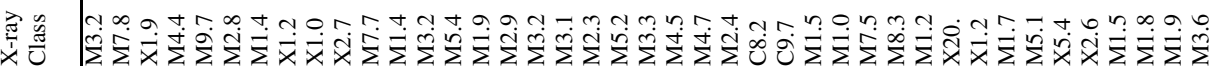

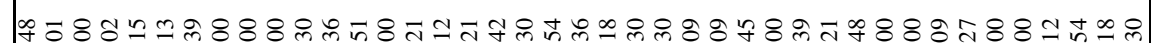

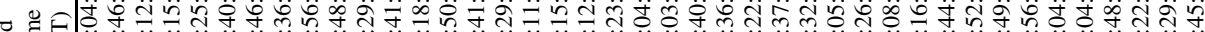

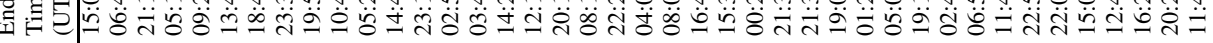

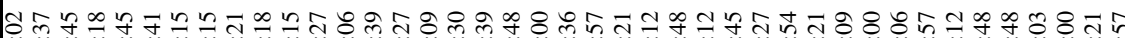

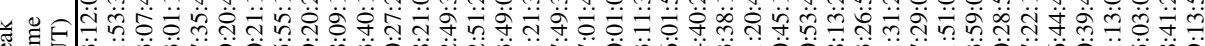

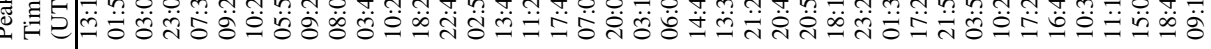

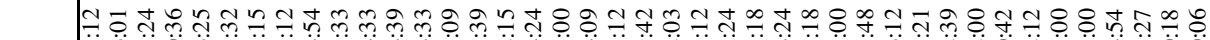
—

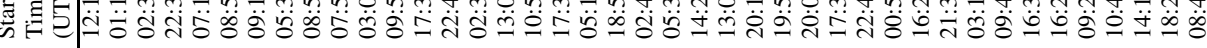

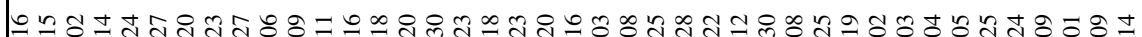

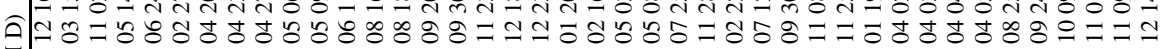

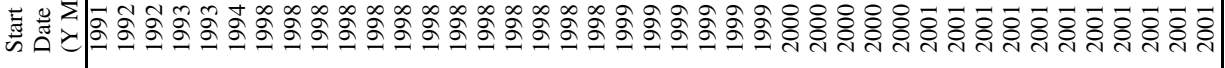

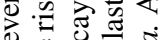

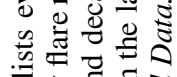
ঊ

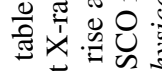

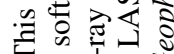
. 政心 - $\dot{0}$ 


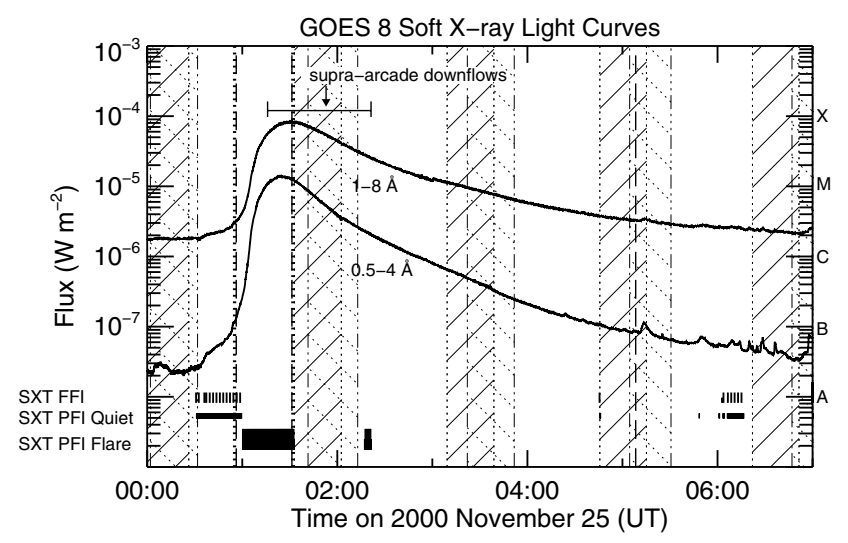

Fig. 1. The observed soft X-ray fluxes for the low energy channel ( 1-8 $\AA$ ) (the curve with the higher flux) and the high energy channel ( $\sim 0.5-4 \AA$ ) (the lower flux) from the detectors on the GOES 8 satellite for the flare on 2000 November 25. Solid-hatched intervals denote Yohkoh night; dotted-hatched intervals indicate Yohkoh passage through the South Atlantic Anomaly region. The times of Yohkoh SXT observations are indicated by small vertical tick marks which sometimes appear as bands. The dotted lines indicate the interval of observed hard X-ray bursts, while the dashed lines indicate the estimated GOES soft X-ray flare start, peak, and end.

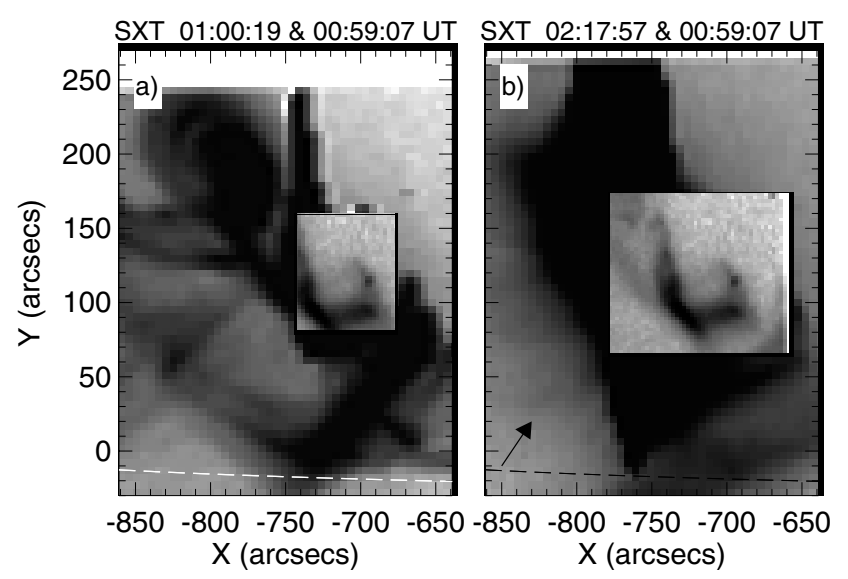

Fig. 2. Portions of SXT half-resolution (4.91" pixel) images with superposed full-resolution $\left(2.45^{\prime \prime}\right.$ pixel) images for the event on 2000 November 25 . The times of each, respectively, are given at the top of each panel. a) shows the soft X-ray structures before the supraarcade downflow are seen, while b) shows an image later in the flare where the supra-arcade downflows (indicated by the black arrow) are clearly seen (in movies of the data).

loops are observed. Figure $2 \mathrm{~b}$ shows an SXT image of the supraarcade late in the event when they are clearly visible (in movies of the data). For this event we find that the supra-arcade downflows as indicated by the proxy of waving coronal rays occurred during the interval of the rise and decay phases of the GOES soft X-ray flare. Moreover, this event was associated with clear erupting loops structures seen in soft X-ray images prior to the appearance of the supra-arcade downflows.

The BATSE DISCLA and HXT data indicate hard X-ray bursts from 00:55:09-01:31:55 UT. These are shown in Fig. 3 where we also show the estimated intervals of the supra-arcade downflows as indicated by the waving coronal rays. The graph shows that the interval during which waving coronal rays were observed overlaps the interval of hard X-ray bursts.

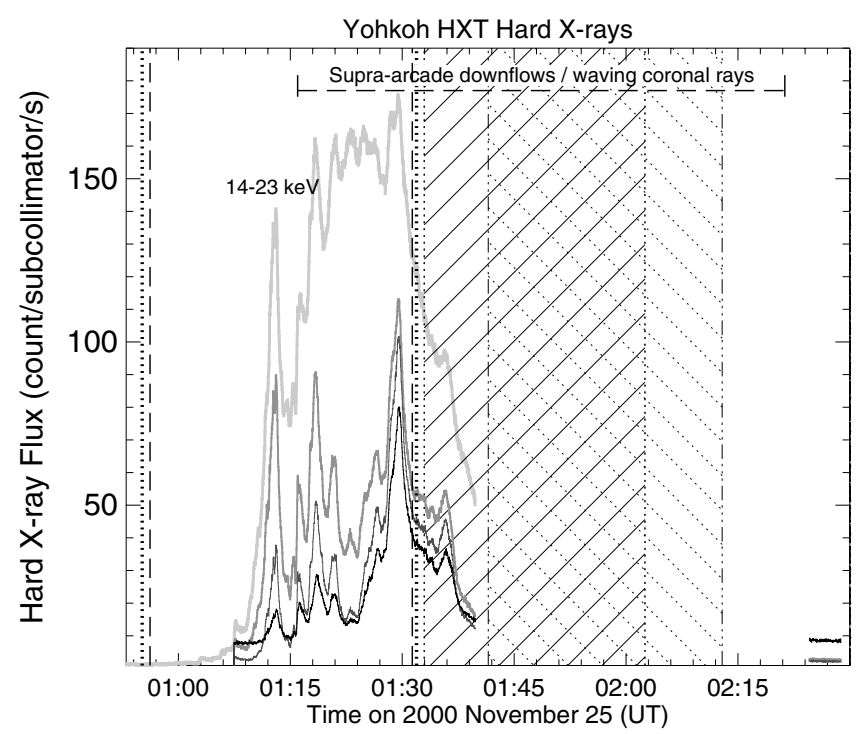

Fig. 3. The observed fluxes from the four channels on the Yohkoh HXT for the flare on 2000 November 25. The solid-hatched interval denotes Yohkoh night; the dotted-hatched interval indicates Yohkoh passage through the South Atlantic Anomaly region. Note the time of observed supra-arcade downflows overlaps the interval of observed hard $\mathrm{X}$-rays. The dotted lines indicate the estimated interval of observed hard $\mathrm{X}$-ray bursts, while the dashed lines indicate the estimated GOES soft $\mathrm{X}$-ray flare start and peak.

\subsection{Downflows starting during the flare decay phase}

We find only one event where the supra-arcade downflows clearly started during the flare decay phase. This is the event of 1998 May 06. This flare has a variety of interesting phenomena, some of which have been examined and reported elsewhere (Khan et al. 2000; Klassen et al. 2001; Hudson et al. 2003; Khan et al. 2006). In particular, Khan et al. (2006) found simultaneous retracting and expanding soft X-ray emitting coronal loops above the main flaring arcade during the decay phase of the flare. The supra-arcade downflows appeared shortly after these moving loops. The GOES soft X-ray flux for this flare is shown in Fig. 4. It can be seen that SXT observed the whole of the rise phase of this flare, the start of the decay phase, then there is a data gap due to Yohkoh satellite night, after which it observed later in the decay phase also in flare-mode, and later still in quietmode.

Careful examination of all of the SXT flare-mode and quietmode PFI data shows no evidence for supra-arcade downflows or a fan of coronal rays during the flare rise phase. When SXT images are taken after the satellite night interval after the peak of the flare we observe a (non-spiny) "smooth" flaring arcade from $\sim 08: 58: 30$ UT. We estimate this changes to a spiny arcade at 09:08 UT. We estimate the waving coronal rays occur between $\sim 09: 11: 20-09: 52: 56$ UT. Similar to Fig. 2, we show images before the supra-arcade downflows are seen and late in the event when the coronal rays are clearly visible. This event unambiguously shows supra-arcade downflows (or laterally moving coronal rays) starting during the flare decay phase. Note that the time of the start of the supra-arcade downflows is near a change in gradient of the GOES soft X-ray flux (see Fig. 4). This may indicate additional energy input late in the flare.

The HXT data for this flare show hard X-ray bursts which, as well as occurring during the flare rise phase, also occur later. These hard X-ray bursts temporally overlap part of the supraarcade downflow interval as inferred from the proxy of waving 


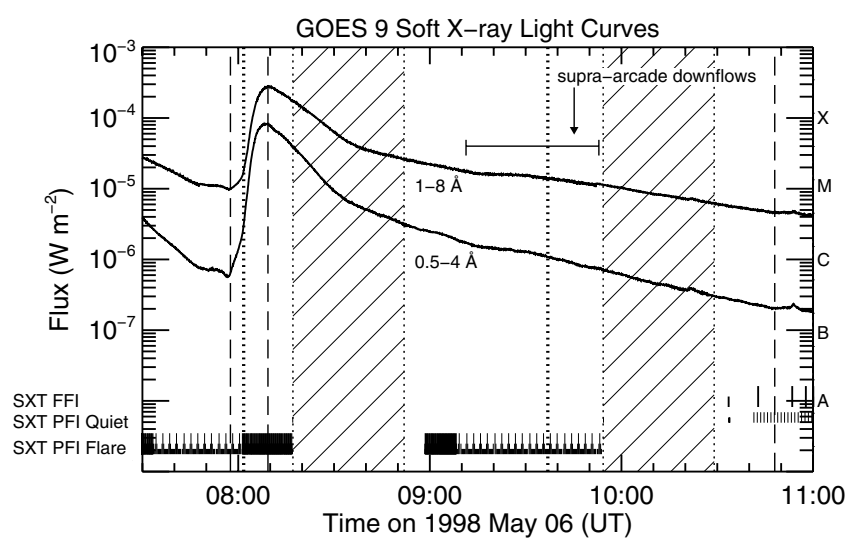

Fig. 4. The observed soft X-ray fluxes for the low energy channel $(\sim 1-8 \AA$ ) (the curve with the higher flux) and the high energy channel $(\sim 0.5-4 \AA$ ) (the lower flux) from the detectors on the GOES 9 satellite for the flare on 1998 May 06. Solid-hatched intervals denote Yohkoh night. The times of Yohkoh SXT observations are indicated by small vertical tick marks which sometimes appear as bands. The dotted lines indicate the interval of observed hard X-ray bursts, while the dashed lines indicate the estimated GOES soft X-ray flare start, peak, and end.
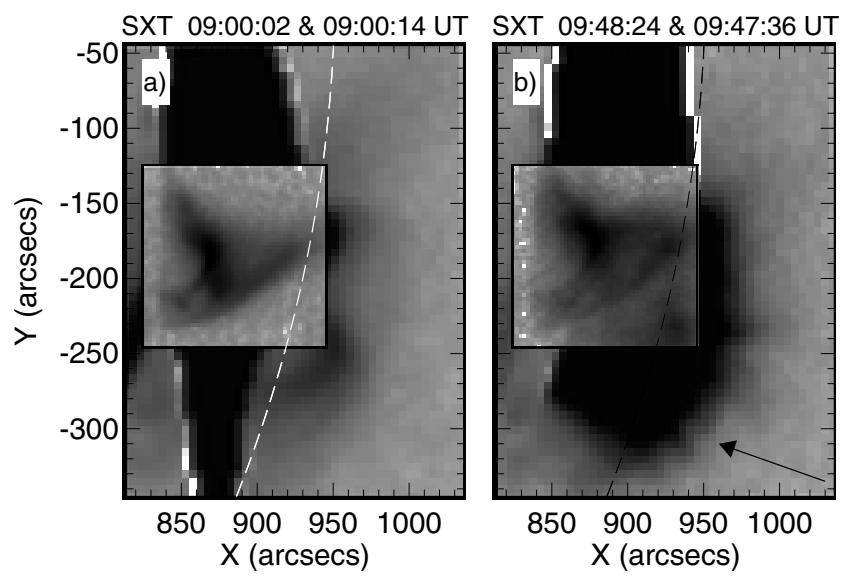

Fig. 5. Portions of SXT half-resolution (4.91" pixel) images with superposed full-resolution $\left(2.45^{\prime \prime}\right.$ pixel) images for the event on 1998 May 06. The times of each, respectively, are given at the top of each panel. a) shows the soft X-ray structures before the supra-arcade downflow are seen, while b) shows an image later in the flare where the supra-arcade downflows (indicated by the black arrow) are clearly seen (in movies of the data).

coronal rays. The HXT data and the interval of waving coronal ray are shown in Fig. 6. It should be noted that although the supra-arcade downflows occur late in the flare they do occur during the times of hard X-ray bursts. While this is not the interval of most intense hard X-ray emission, the time interval 09:14-09:30 UT shows small hard X-ray bursts that are observed in all of the HXT channels. (Note, the enhanced flux in the 53-93 keV channel after 09:30 UT is of non-solar origin).

\subsection{Supra-arcade downflows with uncertain start times}

We now illustrate an example of an event with poor temporal coverage which means we are uncertain when the supra-arcade downflows started and whether they overlapped the soft X-ray flare rise phase.

We consider the event on 1993 June 24. The GOES soft $\mathrm{X}$-ray flux for this flare is shown in Fig. 7. From this figure it can

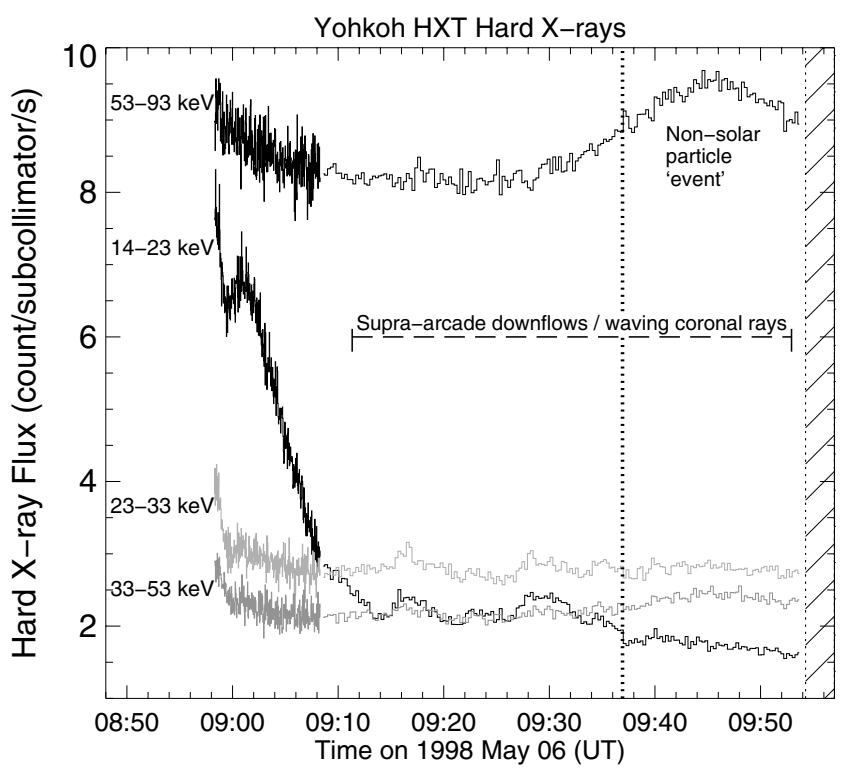

Fig. 6. The observed fluxes from the channels on the Yohkoh HXT for the flare on 1998 May 06. The solid-hatched interval denotes Yohkoh night. Note the time of observed supra-arcade downflows overlaps the interval of observed hard X-rays. The dotted line indicate the end of the observed hard X-ray bursts. Note the increase in the $53-93 \mathrm{keV}$ channel from 09:30 UT onwards is non-solar in origin and should be ignored here.

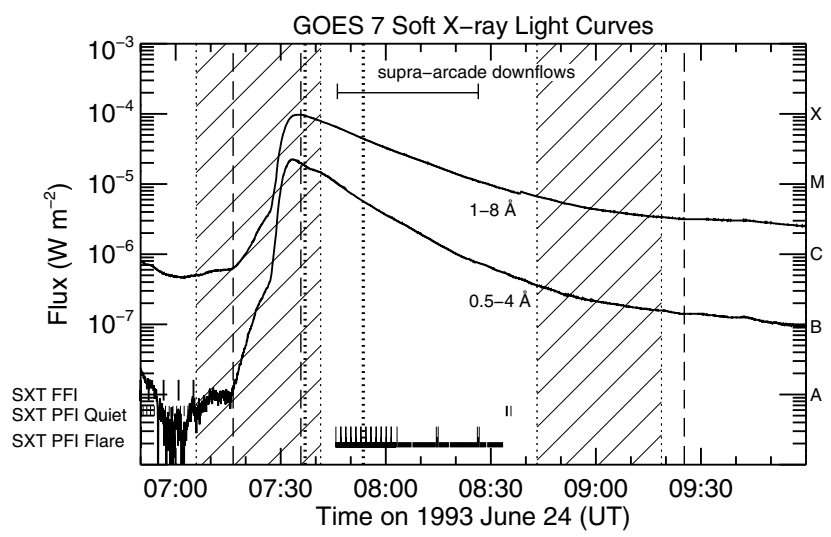

Fig. 7. The observed soft X-ray fluxes for the low energy channel ( $\sim 1-8 \AA$ ) (the curve with the higher flux) and the high energy channel ( $\sim 0.5-4 \AA$ ) (the lower flux) from the detectors on the GOES 7 satellite for the flare on 1993 June 24. Solid-hatched intervals denote Yohkoh night. The times of Yohkoh SXT observations are indicated by small vertical tick marks which sometimes appear as bands. The dotted lines indicate the interval of observed hard X-ray bursts, while the dashed lines indicate the estimated GOES soft X-ray flare start, peak, and end.

be seen that Yohkoh did not observe any part of the rise phase of this flare nor the peak of the flare.

The SXT flare-mode data for this event show supra-arcade downflows (or more accurately waving coronal rays) during the observations after Yohkoh satellite night after the peak of the associated soft X-ray flare. We do not know when these downflows began. It is conceivable that they may have started during the flare rise phase, but we have no observational evidence for or against this suggestion. An SXT image early in the flare and also late in the flare (when the rays are clearly seen) are shown in Fig. 8. In this figure we only show show full-resolution 

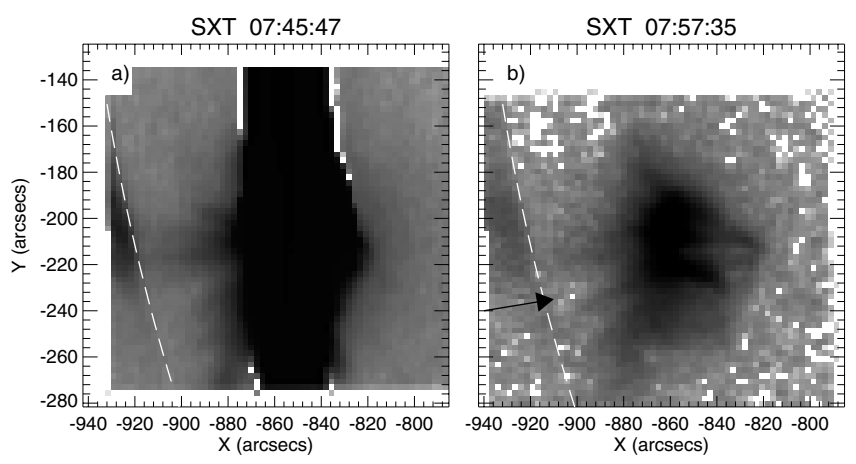

Fig. 8. Portions of SXT full-resolution (2.45" pixel) images for the event on 1993 June 24. The times of each image are given at the top of each panel. a) shows the soft X-ray structures before the supra-arcade downflow are seen, while b) shows an image later in the flare where the supra-arcade downflows (indicated by the black arrow) are clearly seen (in movies of the data).

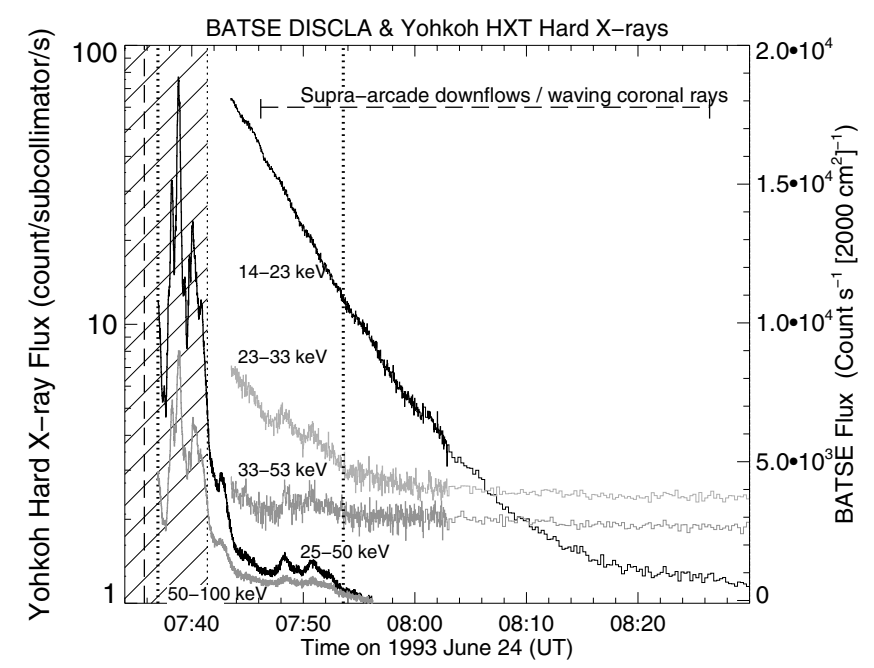

Fig. 9. The observed fluxes from three channels on the Yohkoh HXT and two BATSE DISCLA channels for the flare on 1993 June 24. The solid-hatched interval denotes Yohkoh night. Note the time of observed supra-arcade downflows overlaps the interval of observed hard X-rays. The dotted lines indicate the interval of observed hard X-ray bursts, while the dashed line indicates the estimated time of the GOES soft $\mathrm{X}$-ray peak flux.

(2.45" pixel) images since these were the ones that we used to determine the earliest times of the waving coronal rays.

The hard X-ray data we have examined is shown in Fig. 9. The HXT data shows hard X-ray bursts were already ongoing when Yohkoh emerged from satellite night. The BATSE DISCLA data show there were hard X-ray bursts which occurred earlier. Note that although we clearly observe during the decay phase of the flare hard X-ray bursts are still occurring at that stage. We find that the interval of the hard X-ray bursts temporally overlaps the interval of supra-arcade downflows and waving coronal rays.

\section{Results}

Table 1 lists the supra-arcade downflow events we found in the SXT data and the results of our analysis. This shows our estimated the times of the associated GOES soft X-ray flare and hard X-ray bursts (as seen with HXT or BATSE DISCLA). We estimate these times are accurate to $\sim \pm 10 \mathrm{~s}$. We also examined the SXT data and determined the interval of supraarcade downflows (either directly observed or inferred from a laterally-moving fan of coronal rays). We estimate these times are accurate to $\sim \pm 20 \mathrm{~s}$. From these times we determined whether the supra-arcade downflows occurred during the (GOES) soft Xray flare rise or decay phases or hard X-ray bursts. In addition, we noted any evidence for an eruptive signature associated with the flare and prior to the downflows.

The results of our analysis can be summarised by the following questions and answers:

- Question 1: Do supra-arcade downflows occur during the GOES soft X-ray decay phase?

Answer: Yes: $100 \%$ (41/41)

- Question 2: Do supra-arcade downflows occur during the GOES soft X-ray rise phase?

Answer: Yes: $73 \%$ (30/41), Uncertain: 24\% (10/41), No: $2 \%(1 / 41)$.

- Question 3: Do supra-arcade downflows occur during hard X-ray bursts?

Answer: Yes: 90\% (37/41), Uncertain: 10\% (4/41), No: 0\% (0/41).

- Question 4: Do supra-arcade downflow events show prior eruptive signatures?

Answer: Yes: 73\% (30/41), Uncertain: 27\% (11/41)

Note that the overlap of supra-arcade downflows is higher with the hard X-ray burst interval than with the soft X-ray rise interval, as many events we do not observe the rise phase but find hard X-ray bursts occurring during the decay phase. For Question 3 we considered all supra-arcade downflow events above, including those for which it is uncertain whether the downflows occurred during the soft $\mathrm{X}$-ray rise phase. If instead we consider only those events for which we know the supraarcade downflows occurred during the soft X-ray rise phase (i.e., yes to Question 2) then we get the following answer concerning whether supra-arcade downflows occur during hard X-ray bursts: Yes: 97\% (29/30).

There is a single event (the one discussed in Sect. 3.2) which shows supra-arcade downflows not occurring during the soft $\mathrm{X}$-ray rise phase. We find no other unambiguously negative answers to the other questions. We conclude that when we have good observational data with good temporal coverage the answers to the above four questions are affirmative.

\section{Conclusions and discussion}

1. All supra-arcade downflow events we examined in the Yohkoh SXT data occur during the decay phase of the corresponding soft X-ray flare.

2. The vast majority of supra-arcade downflow events:

(a) start during the rise phase of the corresponding soft $\mathrm{X}$-ray flare;

(b) overlap the time interval of the flare-associated hard X-ray bursts;

(c) have associated prior eruptive signatures.

Most of the supra-arcade downflow events we examined occur during the rise phase of the soft X-ray flare and during hard $\mathrm{X}$-ray bursts. Furthermore, most supra-arcade downflow events showed prior (or inferred prior) eruptive signatures in coronal imaging data. This shows that supra-arcade downflows are associated with ejective/eruptive flares rather than confined flares and indicates a possible association with the flare energy release 
process or the process of energy relaxation immediately afterwards. If the suggestion that supra-arcade downflows are due to retracting reconnected loops is correct, then that, together with the associations we find in this work suggest the downflows may indeed be directly related to the magnetic reconnection process in flares.

Important information is also provided by the single event which shows supra-arcade downflows not occurring during the soft X-ray rise phase. For that event we do, however, find overlapping hard X-ray bursts, suggesting that the downflows may be related to later energy release.

Overall the results of this analysis appear to support the suggestion of Asai et al. (2004) that supra-arcade downflows are associated with the magnetic reconnection process which gives rise to the flare energy release, including accelerated particles which result in hard X-ray emission.

Acknowledgements. The Yohkoh data used here are courtesy of the Institute of Space and Astronautical Science (ISAS), Japan Aerospace eXploration Agency (JAXA). The GOES data are courtesy of the World Data Center A for Solar-Terrestrial Physics, NGDC, NOAA E/GC2, 325 Broadway, Boulder, Colorado 80303, USA. J.I.K. gratefully acknowledges financial support from a Rolling Research Grant from the Science and Technology Facilities Council (STFC) of the UK. H.M.B. gratefully acknowledges an STFC Postgraduate Studentship and financial support from the Dept. of Physics \& Astronomy, University of Glasgow. Financial support by the European Commission through the SOLAIRE Network (MTRN-CT-2006-035484) is gratefully acknowledged.

\section{References}

Asai, A., Yokoyama, T., Shimojo, M., \& Shibata, K. 2004, ApJ, 605, L77 Brueckner G. E., Howard, R. A., Koomen, M. J., et al. 1995, Sol. Phys., 162, 357 Delaboudinière, J.-P., Artzner, G. E., Brunaud, J., et al. 1995, Sol. Phys., 162 291
Domingo, V., Fleck B., \& Poland, A. I. 1995, Sol. Phys., 162, 1

Fishman, G. J., Meegan, C. A., Wilson, R. B., et al. 1989, in Proc. GRO Science

Workshop, ed. W. N. Johnson (Greenbelt: NASA), 2

Forbes, T. G., \& Acton, L. W. 1996, ApJ, 459, 330

Gallagher, P. T., Dennis, B. R., Krucker, S., Schwartz, R. A., \& Tolbert, K. 2002, Sol. Phys., 210, 341

Grechnev, V. V., Uralov, A. M., Zandanov, V. G., et al. 2006, PASJ, 58, 55

Handy, B. N., Acton, L. W., Kankelborg, C. C., et al. 1999, Sol. Phys., 187, 229

Hirayama, T. 1974, Sol. Phys., 34, 323

Hudson, H. S. 2000, A\&A, 531, L75

Hudson, H. S., \& McKenzie, D. E. 2001, Earth Planets Space, 53, 581

Hudson, H. S., Khan, J. I., Lemen, J. R., et al. 2003, Sol. Phys., 212, 121

Innes, D. E., McKenzie, D. E., \& Wang, T. 2003a, Sol. Phys., 217, 247

Innes, D. E., McKenzie, D. E., \& Wang, T. 2003b, Sol. Phys., 217, 267

Khan, J. I., \& Hudson, H. S. 2000, Geophys. Res. Lett., 27, 1083

Khan, J. I., Vilmer, N., Saint-Hilaire, P., \& Benz, A. O. 2002, A\&A, 388, 363

Khan, J. I., Fletcher, L., \& Nitta, N. V. 2006, A\&A, 453, 335

Klassen, A., Aurass, H., \& Mann, G. 2001, A\&A, 370, L41

Kopp, R. A., \& Pneuman, G. W. 1976, Sol. Phys., 50, 85

Kosugi, T., Makishima, K., Murakami, T., et al. 1991, Sol. Phys., 136, 17

McKenzie, D. E. 2000, Sol. Phys., 195, 381

McKenzie, D. E., \& Hudson, H. S. 1999, ApJ, 519, L93

McKenzie, D. E., \& Hudson, H. S. 2001, Earth Planets Space, 53, 577

Ohyama, M., \& Shibata, K. 1997, PASJ, 49, 249

Ohyama, M., \& Shibata, K. 1998, ApJ, 499, 934

Phillips, K. J. H., Chifor, C., \& Landi, E. 2005, ApJ, 626, 1110

Priest, E. R., \& Forbes, T. 2000, Magnetic Reconnection: MHD Theory and Applications (Cambridge: Cambridge University Press)

Sheeley, N. R., Jr., Warren, H. P., \& Wang, Y.-M. 2004, ApJ, 616, 1224

Švestka, Z. F., Fontenla, J. M., Machado, M. E., Martin, S. F., \& Neidig, D. F. 1987, Sol. Phys., 108, 237

Švestka, Z., Fárník, F., Hudson, H. S., \& Hick, P. 1998, Sol. Phys., 182, 179

Tripathi, D., Solanki, S. K., Schwenn, R., et al. 2006, A\&A, 449, 369

Tsuneta, S., Acton, L. W., Bruner, M. E., et al. 1991, Sol. Phys., 136, 37

Verwichte, E., Nakariakov, V. M., \& Cooper, F. C. 2005, A\&A, 430, L65

Wang, J., Shibata, K., Nitta, N., et al. 1997, ApJ, 478, L41

Wang, Y.-M., Sheeley, N. R., Jr., Howard, R. A., St. Cyr, O. C., \& Simnett, G. M. 1999, Geophys. Res. Lett., 26, 1203 